\title{
Mechanical response of two different molecular weight polycarbonates at varying rates and temperatures
}

\author{
Peihao Song ${ }^{1,2^{*}}$, Akash $R$ Trived ${ }^{1,2}$, and Clive $R$ Siviour $^{1}$ \\ ${ }^{1}$ Department of Engineering Science, University of Oxford, Parks Road, OX1 3PJ, UK \\ ${ }^{2}$ DPI, P.O. Box 902, 5600 AX Eindhoven, the Netherlands
}

\begin{abstract}
Polymers are widely used for lightweight design in industrial applications, such as helmets and car bumpers, where the most common causes of failure or damage are dynamic impact events. It is well known that the mechanical response of most polymers is highly dependent on the loading rate and temperature, and that it is not sufficient to use properties measured under static loads in the analysis of dynamic events. However, the time-temperature equivalence phenomenon offers the chance to predict high-rate performance using low-rate data. In this study, information about the constitutive behaviour of two different molecular weight polycarbonates, is obtained in low-rate experiments and then compared with the high-rate response. A master curve of storage modulus constructed from Dynamic Mechanical Analysis data is employed to understand the viscoelastic response under small-strain loading at various frequencies and temperatures. For the large-strain constitutive response, experiments at strain rates from $0.001 \mathrm{~s}^{-1}$ to $3000 \mathrm{~s}^{-1}$ are performed using a conventional crosshead device, hydraulic device, and split-Hopkinson pressure bar. Moreover, experiments at strain rates of $0.01 \mathrm{~s}^{-1}$ and temperatures from -60 to $120{ }^{\circ} \mathrm{C}$ are also performed, and the results are compared. This approach can distinguish 'constitutive' rate dependence from the effects of specimen self-heating due to adiabatic heating under high-rate deformation. Meanwhile, the molecular weight effects on the mechanical response at varying rates and temperatures are also noted.
\end{abstract}

\section{Introduction}

Polycarbonate (PC) is a typical glassy amorphous polymer, and the characterisation of its mechanical response has been investigated for many decades. It is well-known that the mechanical properties of polymers exhibit strong temperature and rate sensitivity, which is expressed as increasing strength and stiffness with decreasing temperature or increasing strain rate [1-4]. Thus, using low rate data is not adequate to analyse the high rate performance of polymer materials. On the other hand, it is challenging to measure the mechanical properties of polymers under dynamic loading because of their low impedance and strength [5]. Moreover, increasing the strain rate can change experimental conditions

* Corresponding author: peihao.song@eng.ox.ac.uk 
from isothermal to adiabatic $[6,7]$. Thus, when investigating the high rate performance of materials, during which the mechanical work of plastic deformation is partly converted to heat, and the temperature rising can induce a qualitatively different constitutive response to that observed in low rate experiments $[8,9]$. In this paper, the time-temperature equivalence theory is applied to analyse the results at different temperatures and rates obtained from two different PCs.

\section{Material information and sample preparation}

Table 1 shows a list of the two different grades of injection moulded PCs used in this study. Two different melt flow rate (MFR) homo-PCs are characterised. A material with a high MFR has a low molecular weight. The materials were obtained as injection moulded sheets of dimensions 175,175 , and $3 \mathrm{~mm}$. To minimise the residual stress resulting from manufacturing, the $\mathrm{PC}$ sheets were annealed at $10^{\circ} \mathrm{C}$ above their glass transition temperature $\left(\mathrm{T}_{\mathrm{g}}\right)$ for six hours, following which the temperature was decreased to room temperature at a $10{ }^{\circ} \mathrm{C} /$ hour cooling rate. The maximum dimension change of the plates was $1.5 \%$ after thermal treatment, and the removal of in-plane residual stress was monitored using images taken through polarising film.

Table 1. Grades of PC samples used in this study.

\begin{tabular}{cccc}
\hline Polymer & Code & Melt Flow Rate (MFR) at $300^{\circ} \mathrm{C} / 1.2 \mathrm{~kg}$ & $\mathrm{~T}_{\mathrm{g}}\left({ }^{\circ} \mathrm{C}\right)$ \\
\hline LEXAN $^{\mathrm{TM}}$ RESIN 103R & PC1 & 6 & 145 \\
LEXAN $^{\mathrm{TM}}$ RESIN HF1110 & PC2 & 25 & 145 \\
\hline
\end{tabular}

All specimens are cut from the centre of the annealed sheets to avoid any remaining residual stress at the edges of the plates. For compression experiments, cylinders with a 5 $\mathrm{mm}$ diameter and a $3 \mathrm{~mm}$ thickness were used. For Dynamic Mechanical Analysis (DMA), experiments with a single cantilever configuration were performed on rectangular samples of $40 \mathrm{~mm}$ length, $10 \mathrm{~mm}$ width and $2 \mathrm{~mm}$ thickness.

\section{Experimental characterisation}

DMA experiments were carried out to measure the frequency and temperature dependence of the mechanical properties in the linear viscoelastic region. Large strain compression experiments were conducted to investigate the constitutive behaviour. The same setup was employed for the two different PCs in all experiments.

\subsection{Dynamic Mechanical Analysis (DMA)}

DMA experiments were conducted on a TA Instruments Q800. An isothermal frequency sweep was used with $0.1 \%$ strain, a temperature range from -110 to $170{ }^{\circ} \mathrm{C}$ with $2{ }^{\circ} \mathrm{C}$ increments and 5 minutes isothermal, and frequencies of $0.5,1,2,5$ and $10 \mathrm{~Hz}$. 


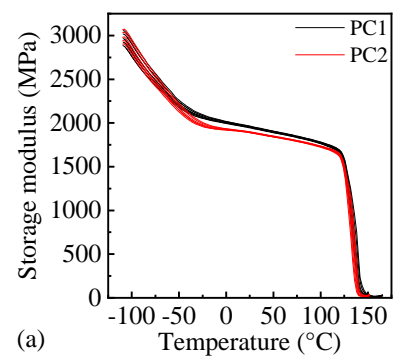

(a)

Temperature $\left({ }^{\circ} \mathrm{C}\right)$
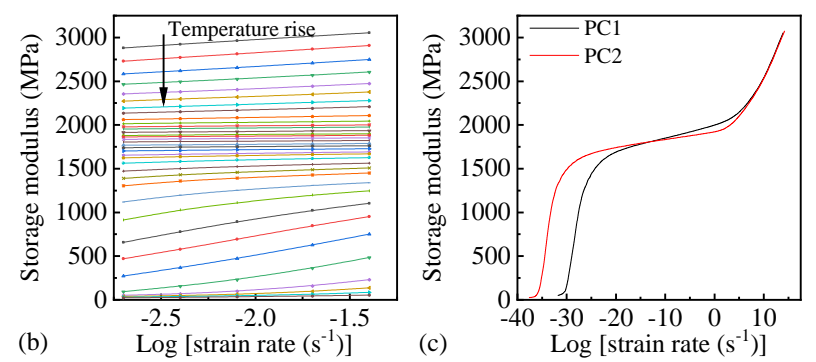

Fig. 1. (a) Storage modulus versus temperature at different frequencies, (b) Isotherms showing the dependence of modulus on frequency and temperature (PC1), (c) Time-temperature superposition curves.

In Fig. 1(a), it can be observed clearly that the moduli are affected by both temperature and frequency. With increasing temperature, the modulus of both PCs decreases gradually until the temperature reaches the glass transition temperature $\left(\mathrm{T}_{\mathrm{g}}\right)$, which there is a very large drop. In particular, the modulus of high molecular weight polycarbonate (PC1) is higher than the low molecular weight polycarbonate (PC2) in the temperature region of -50 to $150{ }^{\circ} \mathrm{C}$, and from -110 to $-40 \mathrm{C}$, the modulus of PC2 shows more frequency-dependence than that of PC1.

It is noted that the transition temperature increases with increasing frequency. This property of polymers forms the base of time-temperature superposition (TTS), in which the effect of increasing the frequency is similar to that of decreasing the temperature and viceversa. In order to perform approximate comparisons to the later compression data, the frequency in the DMA test is converted to an approximate strain rate using

$$
\dot{\varepsilon} \approx \frac{\Delta \varepsilon}{\Delta t}=\frac{\varepsilon_{0}}{1 / 4 f}=4 f \varepsilon_{0}
$$

where $f$ and $\varepsilon_{0}$ are applied frequencies and strain in the DMA experiments. Instead of plotting the storage modulus against temperature, Fig. 1(b) presents the moduli versus the $\log$ strain rate of PC1 as an example; for clarity, not all of the isotherms obtained are shown. The basic principle of the horizontal shifting algorithm is that the isotherms at temperatures above the reference temperature $\left(20^{\circ} \mathrm{C}\right)$ are shifted to the left-hand side (i.e. lower frequency), the isotherms below the reference temperature are moved to the right-hand side (higher frequency), so that each segment overlaps with the previous. There are many methods for overlapping the isotherms, which can result in slightly different final master curves. In this current work, manual shifting is used to obtain the master curves as shown in Fig. 1(c); the details of this method can be seen in previously published work $[9,10]$.

\subsection{Varying temperature and strain rate compression tests}

The quasi-static (QS) compressive response at controlled true strain rates of $0.001 \mathrm{~s}^{-1}, 0.01 \mathrm{~s}^{-}$ ${ }^{1}$ and $0.1 \mathrm{~s}^{-1}$ was obtained using an Instron 5980 universal test machine. For the true strain rate controlled $\left(0.01 \mathrm{~s}^{-1}\right)$ temperature-dependent characterisation, the temperature is maintained using an environmental chamber equipped with a convection heater and liquid nitrogen from $-60{ }^{\circ} \mathrm{C}$ to $120^{\circ} \mathrm{C}$. The medium rate $\left(5\right.$ to $\left.50 \mathrm{~s}^{-1}\right)$ and high rate $\left(1000\right.$ to $3000 \mathrm{~s}^{-}$ $\left.{ }^{1}\right)$ experiments are conducted using a hydraulic machine and split Hopkinson pressure bar (SHPB), respectively. To avoid friction effects [11], petroleum jelly was used as a lubricant at room temperature and above, AEROSPEC ${ }^{\circledR} 210$ was employed for low-temperature tests. 

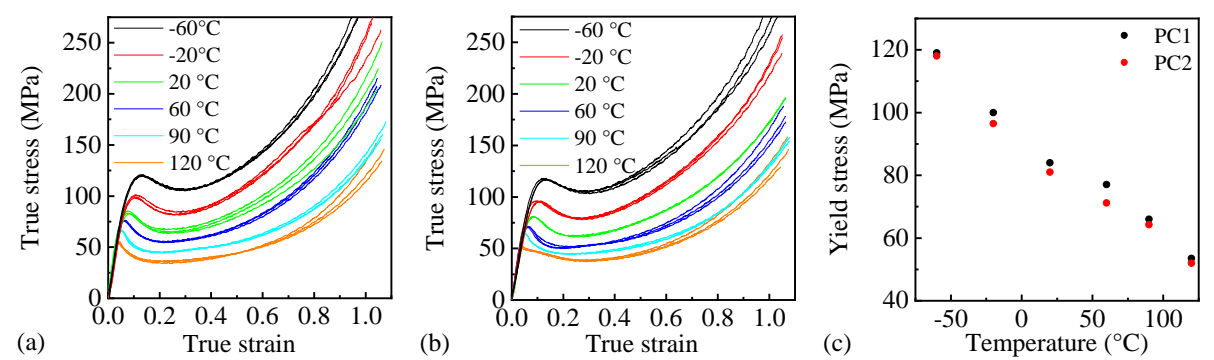

Fig. 2. (a) $P C 1$ stress-strain curve at $0.01 \mathrm{~s}^{-1}$ and varying temperatures, (b) $P C 2$ stress-strain curve at $0.01 \mathrm{~s}^{-1}$ and varying temperatures, (c) Temperature-dependent yield stress comparing between PC1 and PC2.

Fig. 2 shows results from experiments conducted at varying temperatures from $-60{ }^{\circ} \mathrm{C}$ to $120^{\circ} \mathrm{C}$ and a constant true strain rate of $0.01 \mathrm{~s}^{-1}$. For both PCs, with decreasing temperature, the strength increases, and vice versa (Fig. 2(c)). Moreover, the trend of the yield stress of PCs is consistent with DMA results that high molecular weight PC is stronger than the one with low molecular weight when temperature higher than $-50^{\circ} \mathrm{C}$, and at $-60^{\circ} \mathrm{C}$, the yield stresses of the different PCs are very close to each other.
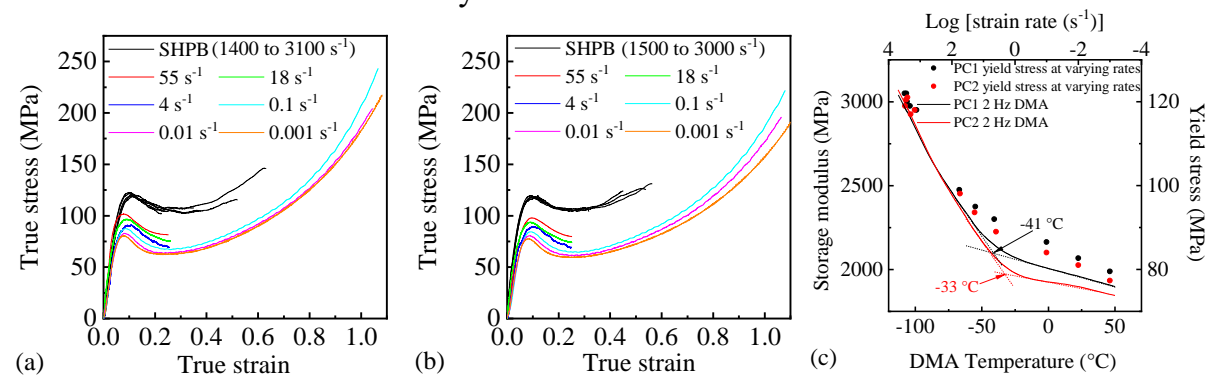

Fig. 3. (a) PC1 stress-strain curve at varying rates and room temperature, (b) PC2 stress-strain curve varying rates and room temperature, (c) Rate-dependent yield stress and $2 \mathrm{~Hz}$ DMA result comparison for PC1 and PC2.

The results of varying rate tests at room temperature $\left(20^{\circ} \mathrm{C}\right)$ are plotted together in Fig. 3(a) and Fig. 3(b) for PC1 and PC2. The yield stress increases with increasing strain rate, and, in Fig. 3(c), the yield stress shows a more rapid increase with the logarithm of strain rate at the higher rates; these data are obtained from the SHPB test. Furthermore, the compression yield stress is again consistent with DMA data, also presented in 3(c). With increasing strain rate or decreasing temperature, the PCs become stronger and stiffer, probably as a result of the beta transition. In Fig. 3(c), the inflection point corresponding to this transition on the 2 $\mathrm{Hz}$ DMA data of $\mathrm{PC} 1$ and $\mathrm{PC} 2$ are at $-41{ }^{\circ} \mathrm{C}$ and $-33{ }^{\circ} \mathrm{C}$, respectively, i.e. it is a higher temperature for the lower molecular weight. Equivalently, for the same polymer, the inflection in the yield stress occurs at a lower strain rate.

\section{Discussion}

Fig. 4(a) shows the comparison between strain rate- and the temperature-dependent yield stress: increasing strain rate is equivalent to decreasing the temperature, this is the so-called time-temperature equivalence which has been reported in the literature [8]. 


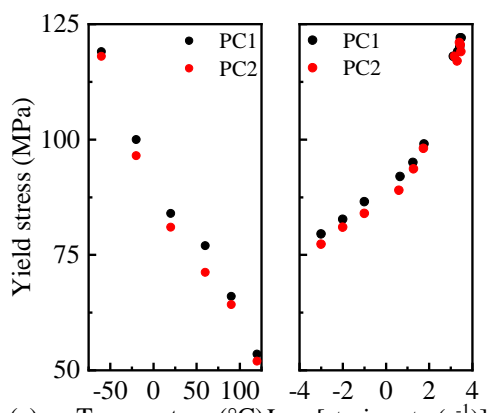

(a) Temperature $\left({ }^{\circ} \mathrm{C}\right) \log \left[\right.$ strain rate $\left.\left(\mathrm{s}^{-1}\right)\right]$

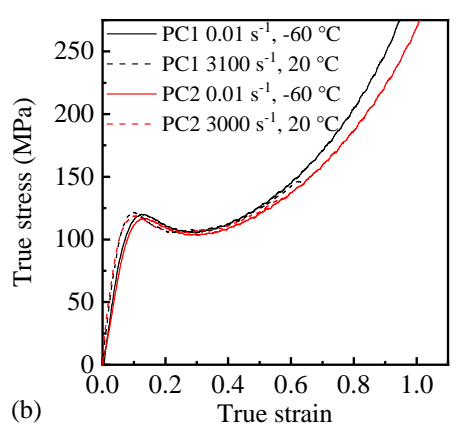

Fig. 4. (a) Yield stress versus temperature and yield stress versus log strain rate of PC1 and PC2 (b) Comparing large strain behaviour between low temperature quasi-static and high rate room temperature tests on PC1 and PC2.

For the large strain behaviour, the effects of adiabatic heating are considered for high rate loading, and the following equations were employed to estimate at which strain rate the transition from isothermal to adiabatic heating occurs in PCs

$$
\begin{aligned}
\alpha & =\frac{k}{\rho C_{p}} \\
l_{t} & =2 \sqrt{\alpha t} \rightarrow t=\frac{1}{\alpha}\left(\frac{l_{t}}{2}\right)^{2} \\
\dot{\varepsilon} & =\frac{1}{t}
\end{aligned}
$$

Here the thermal diffusivity $\alpha$ is calculated from the thermal conductivity, $k\left(0.2 \mathrm{~W} \mathrm{~m}^{-1}\right.$ $\left.\mathrm{K}^{-1}\right)$, density $\rho\left(1.2 \mathrm{~g} \mathrm{~cm}^{-3}\right)$, and specific heat capacity $C_{P}\left(1.2 \mathrm{~J} \mathrm{~g}^{-1} \mathrm{~K}^{-1}\right)$ using Equation (2). Following that, Equation (3) is using to estimate the timescale for thermal diffusion $t$, here $l_{t}$ is the length of the specimen. Using this method, the transition from isothermal to adiabatic conditions starts at a strain rate on the order of $0.6 \mathrm{~s}^{-1}$. Experiments at $0.01 \mathrm{~s}^{-1}$ with a temperature of $-60{ }^{\circ} \mathrm{C}$ and a high rate test at $3000 \mathrm{~s}^{-1}$ and room temperature are plotted in Fig. 4(b). This figure shows that the constitutive behaviour of the low-temperature QS test and room temperature high rate test is similar to each other at strains up to 0.55 . This is consistent with the results of Kendall and Siviour [1], but is still surprising. Significant temperature rises in high rate loading of PC have been reported in the literature [6], but the data here imply that the temperature rise does not qualitatively affect the stress-strain relationship in the material.

\section{Conclusion}

The paper shows results from DMA experiments and varying rate and temperature compressive experiments for two different molecular weight polycarbonates. The timetemperature equivalence theory performs well when comparing low temperature and high rate test results for both PCs in a small strain regime. DMA results show a good consistency with the mechanical test results. Moreover, the yield stress at the strain rate of above $10 \mathrm{~s}^{-1}$ appears to converge for the different grade PCs. Similarly, the yield stresses of these two material overlap at the lowest temperature and the converge of modulus is also observed in the DMA result at low temperature and master curve at high rate region, which indirectly enhances the time-temperature equivalence theory. 
For the large strain deformation behaviours, although the temperature significantly rises in high rate loading, the strain softening and strain hardening behaviours are not affected compared to the quasi-static results. This indicates the low rate low temperature data can be used to represent high strain rate behaviour; however, further work is needed to fully understand the mechanisms that govern post-yield responses and this apparent lack of sensitivity.

This research forms part of the research programme of DPI, project 827t19, Impact Modelling of Polymers: high-Rate Experiments for Solid-state Simulations. The authors thank our colleagues on this project, Dr Davide De Focatiis and Grace Owen at the University of Nottingham for helpful discussions. We also thank the technicians of the Solid Mechanics Group workshop, in particular Andy Bateman and Richard Duffin, for their help with the manufacture of specimens and fixtures for this project.

\section{Reference}

1. Kendall, M.J. and C.R. Siviour, Experimentally simulating high-rate behaviour: rate and temperature effects in polycarbonate and PMMA. Philos Trans A Math Phys Eng Sci, 2014. 372(2015): p. 20130202.

2. Siviour, C.R., et al., The high strain rate compressive behaviour of polycarbonate and polyvinylidene difluoride. Polymer, 2005. 46(26): p. 12546-12555.

3. Richeton, J., et al., Influence of temperature and strain rate on the mechanical behavior of three amorphous polymers: Characterization and modeling of the compressive yield stress. International Journal of Solids and Structures, 2006. 43(78): p. 2318-2335.

4. Mulliken, A.D. and M.C. Boyce, Mechanics of the rate-dependent elastic-plastic deformation of glassy polymers from low to high strain rates. International Journal of Solids and Structures, 2006. 43(5): p. 1331-1356.

5. Siviour, C.R. and J.L. Jordan, High Strain Rate Mechanics of Polymers: A Review. Journal of Dynamic Behavior of Materials, 2016. 2(1): p. 15-32.

6. Rittel, D., On the conversion of plastic work to heat during high strain rate deformation of glassy polymers. Mechanics of Materials, 1999. 31(2): p. 131-139.

7. Sorini, C., A. Chattopadhyay, and R.K. Goldberg, Micromechanical modeling of the effects of adiabatic heating on the high strain rate deformation of polymer matrix composites. Composite Structures, 2019. 215: p. 377-384.

8. Kendall, M.J. and C.R. Siviour, Experimentally simulating adiabatic conditions: Approximating high rate polymer behavior using low rate experiments with temperature profiles. Polymer, 2013. 54(18): p. 5058-5063.

9. Trivedi, A.R. and C.R. Siviour, A novel methodology for predicting the high rate mechanical response of polymers from low rate data: application to (plasticised) poly(vinyl chloride). Mechanics of Time-Dependent Materials, 2020.

10. Yoon, S.-h., I. Giannakopoulos, and C.R. Siviour, Application of the Virtual Fields Method to the uniaxial behavior of rubbers at medium strain rates. International Journal of Solids and Structures, 2015. 69: p. 553-568.

11. Trautmann, A., et al., Lubrication of polycarbonate at cryogenic temperatures in the split Hopkinson pressure bar. International Journal of Impact Engineering, 2005. 31(5): p. 523-544. 